\title{
Les arts au creuset de la pensée congolaise contemporaine
}

The Arts in the Crucible of Contemporary Congolese Thought

Jean-Luc Aka-Evy

\section{(2) OpenEdition}

\section{Journals}

Édition électronique

URL : https://journals.openedition.org/etudesafricaines/16564

DOI : 10.4000/etudesafricaines. 16564

ISSN : 1777-5353

Éditeur

Éditions de l'EHESS

\section{Édition imprimée}

Date de publication : 20 novembre 2010

Pagination : 1215-1240

ISBN : 978-2-7132-2252-8

ISSN : 0008-0055

Référence électronique

Jean-Luc Aka-Evy, «Les arts au creuset de la pensée congolaise contemporaine », Cahiers d'études africaines [En ligne], 198-199-200 | 2010, mis en ligne le 02 janvier 2013, consulté le 21 avril 2022. URL : http://journals.openedition.org/etudesafricaines/16564; DOI : https://doi.org/10.4000/ etudesafricaines. 16564

Ce document a été généré automatiquement le 21 avril 2022.

(c) Cahiers d'Études africaines 


\section{Les arts au creuset de la pensée congolaise contemporaine}

The Arts in the Crucible of Contemporary Congolese Thought

Jean-Luc Aka-Evy

1 Dans le dégagement de la pensée africaine contemporaine à l'épreuve des combats des Noirs-Africains pour les indépendances de leur pays, le travail créatif des artistes (Diop 1951), des écrivains et des intellectuels a été déterminant dans la prise de conscience historique, culturelle et politique des peuples d'Afrique noire. Dès la fin de la Seconde Guerre mondiale, la lutte pour la reconnaissance des héritages culturels et historiques (Diop 1954) du monde noir s'amorce partout en Afrique, en Europe, aux Amériques et dans les Caraïbes, surtout après l'effondrement et la libération du monde occidental (Spengler 1948) suite aux confrontations des deux guerres mondiales. Les prises de position radicales concernant le vécu noir africain se font entendre dans les revues, dans les manifestations culturelles et artistiques internationales (Présence Africaine 1956, 1959) de grande envergure, dans les universités : des publications majeures sur le monde noir sont annoncées et bouleversent la conscience mondiale sur les faits africains. Dès lors, les Noirs-Africains relisent autrement leur histoire et déploient désormais leur pensée suivant leurs propres paramètres (Diop 1967 ; Obenga 1973). On s'affranchit alors d'une certaine épistémologie européocentriste et africaniste pour appréhender le « vrai » monde noir. Des paradigmes nouveaux, aiguisés souvent par les Noirs eux-mêmes s'émancipent et s'autorisent de prendre congé de ceux que l'Occident avait élaborés pour lire et dire le vécu noir-africain. Au modèle gréco-latin, réactivé par les philosophies "providentialistes » de l'histoire des Lumières et du XIX ${ }^{\mathrm{e}}$ siècle, en passant par la révolution copernico-galiléo-newtonienne, le fameux cogito cartésien ainsi que le calcul différentiel de Leibniz, modèle qui s'était instauré comme étant le seul paradigme d'appréhension et d'appréciation des êtres et des choses, il faut désormais compter avec les propositions du modèle négro-africain élaboré et conçu par les artistes, écrivains et intellectuels noirs dans la plupart de leurs travaux au croisement des années 1940 et 1950. 
2 Et s'il est entendu que la plupart des intellectuels africains et de la diaspora prennent le parti pris de faire l'inventaire historique de leur pensée et d'exercer leur droit d'initiative créatrice, c'est qu'ils se donnent également les moyens et les instruments pour pouvoir diffuser et installer leurs pensées. Parmi les revues scientifiques qui vont donner une véritable visibilité au vécu du monde noir et participer à l'élaboration d'une nouvelle vision anthropologique de ce monde, figurent les Cahiers d'Études africaines. La naissance de cette revue dans la droite ligne d'installer le quotidien africain et celui de la diaspora dans l'écriture de la pensée anthropologique moderne, annonce également un remembrement épistémologique de l'approche scientifique du fait culturel africain contemporain. Nous voulons donc, à l'occasion des cinquante ans des Indépendances de quelques pays d'Afrique noire et des Cahiers, amorcer une réflexion sur l'impact des arts urbains dans le déploiement de la pensée congolaise contemporaine.

3 Face donc à cette nouvelle installation de la "pensée africaine " sous toutes ses coutures, face à cette «nouvelle mondialisation » dont le concept «Art nègre » est le signe emblématique, raccordé aux idées novatrices du mouvement panafricaniste qui a érodé en profondeur les Indépendances des pays d'Afrique noire, quel «bilan» historique et esthétique peut-on tirer du travail créatif des artistes d'Afrique noire en général et du Congo en particulier? Quelles sont les résonances des arts dans le mouvement d'émergence du Congo à l'indépendance? Autrement dit, suivant la cadence historique et les combats des forces vives pour la conquête de l'indépendance de notre pays, quel a été le parcours de nos arts et quelles sont les grandes tendances qui marquent l'évolution historique et esthétique de ces arts?

\section{Considérations historiques sur les tendances artistiques du Congo à l'orée des indépendances africaines}

4 Le nom colonial du pays était "Moyen-Congo », c'est-à-dire le pays situé sur le cours moyen du fleuve Congo. Il y avait par conséquent, selon le parcours du fleuve, le BasCongo (pays situés de part et d'autre du cours du fleuve), et le Haut-Congo (contrées localisées dans le cours supérieur du fleuve). La locution «Congo » vient du nom du Royaume de Kongo, localisé dans le nord-ouest de l'Angola actuel (Obenga 1974 : 15-93). Les migrations précoloniales ont amené certains groupes de ce royaume vers le cours inférieur et moyen du fleuve : les Ntandu et les Ndimbu de l'actuel Congo démocratique (ex-Zaïre), les Kongo ou Bakongo, Sundi et Manyanga du Congo-Brazzaville, les Soyo de l'enclave du Cabinda. Les Kongo de Brazzaville disent bien en signe de conscience de leur origine ethnique : "Mase meto ku kongo dia Ntotila batuka " ("Nos ancêtres sont issus du Kongo du chef suprême "ou Roi". Ce chef suprême régnait sur l'ensemble du pays Kongo »). L'orthographe Kongo (Fu-Kiau Kia Bunseki-Lumanisa 1969), c'est pour désigner l'ancien royaume et un groupe humain de ce royaume désormais fixé dans le cours inférieur et moyen du fleuve Nzadi en langue des Kongo, d'où Zaïre dans la phonation portugaise. Car, suivant la traversée historique du Congo, c'est par l'Océan Atlantique, dès 1492, via l'Angola, le San Salvador, et Punta Négra (Pointe-Noire), que le Portugal a pris pied et langue avec le Congo (Proyart $1776: 124-126)$.

5 Par l'Océan Atlantique donc, tout au début de l'ère moderne européenne, par le biais de «l'humanisme» des affaires et des "découvertes» au temps de la Renaissance, le Congo entrait dans les circuits de la mondialisation ibérique et lusitanienne et s'ouvrait 
culturellement au monde (Aka-Evy 2006). À la fin du Xve siècle, les premiers artefacts et objets d'art des Royaumes Kongo et Loango arrivent au Portugal. Ainsi le Congo moderne apparaît dans l'ouvrage d'olfert Dapper (1686: 364-368), publié en néerlandais, en 1668, à Amsterdam. Il y décrit les royaumes du Kongo, du Kakongo, de Soyo, de Loango et de Makoko, en insistant sur les relations économiques, commerciales, artistiques, symboliques et culturelles entre le littoral maritime et le Pool Malébo, c'est-à-dire entre les côtes vili et les savanes téké (ibid. : 59) en passant par la verdoyante vallée du Niari. Les gens des environs de Ibiti (Sibiti de nos jours) chassaient l'éléphant pour le compte des rois-dieux de Loango dont la garde personnelle était recrutée parmi les jeunes valeureux des contrées yaka. Dapper affirme également que les Sundi mélangés aux Téké partaient extraire le cuivre dans la zone qui s'étend de Mindouli à Mouku Songo ou Boko Songo dont l'onomastique signifie précisément «Place du cuivre ». Les affaires, les industries culturelles de l'époque, notamment la métallurgie, le tissage et la poterie, la vaillance de la jeunesse, ont donc constitué le «moteur de l'histoire » et de la culture, pendant des siècles, pour ces pays de savanes et des forêts, de collines et de vallées que l'on admire toujours, entre la côte atlantique et le Congo, jusqu'à son haut bassin, chez les "gens d'eau », les Ngala du Nord Congo. Olfert Dapper a bien noté les arts et les rites de tous ces pays kongo, vili, téké, sundi et ngala, grâce aux informations des marchands négriers des Flandres et des Pays-Bas.

6 Ce qui a sans doute frappé l'imaginaire d'olfert Dapper, ce sont les personnages figurés en estampe dans son ouvrage, il y a trois siècles: ils sont grands, robustes, resplendissants de santé et de vigueur, - une belle race négro-africaine, bien nourrie et bien vêtue, certainement aussi bien soignée, vivant dans des écosystèmes non pollués, selon des rythmes bio-cosmiques et socioculturels appropriés, déroulant ainsi un développement humain harmonieux. Les valeurs culturelles et politiques prônées étaient celles de la solidarité, de la concorde, de la dispute consensuelle, de la palabre négociée sans malice, du respect des ancêtres et des aînés, du culte des morts, de la crainte du père, de l'amour quasi religieux de la mère, du culte du beau, du bien et de la justice (Obenga 1991). La vie individuelle et collective était respectée sans inutiles tautologies. La paix ne se réduisait pas au poids d'un simple slogan, mais elle constituait fondamentalement une valeur de société et de civilisation. Ainsi donc, tout regard tourné vers le passé, même le plus lointain, reste nécessairement soutenu par des interrogations et considérations actuelles puisqu'il s'agit d'une nécessité dialectique et que toute histoire est histoire culturelle contemporaine. En conséquence de quoi, toute politique est d'abord et simplement la traduction d'une politique culturelle arrimée au travail de mémoire d'une civilisation, d'un peuple ou d'une nation. De facto, le plus haut point de la culture d'un peuple consiste à saisir l'esprit universel dans son génie. Pour toutes ces raisons, le domaine culturel doit être un prétexte privilégié pour servir de guide à la découverte et à la connaissance du Congo et de la politique culturelle dégagée par ce pays depuis quelques décennies.

7 En effet, depuis l'amorce de l'indépendance du Congo, la place et le rôle de la culture dans le développement global de ce pays, n'ont jamais été clairement définis par les décideurs et le législateur congolais. La marginalisation permanente du secteur culturel, la faiblesse des investissements, ainsi que le manque des infrastructures qu'on y observe en sont la parfaite illustration. Or, l'histoire des peuples enseigne que la culture consolide la volonté de vivre et d'œuvrer ensemble à la construction d'un pays. La culture contribue donc à développer et à consolider la conscience d'appartenance 
commune à un même pays. Dès lors, la présente étude a pour but de baliser certains repères historiques d'ordres culturel et artistique en vue de servir modestement et éventuellement à l'élaboration d'une politique culturelle efficace, ou tout au moins à la susciter. Étant donné que toute investigation dans ce domaine doit être assortie de principes directeurs clairs reposant sur des pratiques culturelles et artistiques affirmées dans la conscience historique et politique des Congolais.

De fait, notre étude se répartit en deux grandes articulations dont la première partie est un rappel historique de la dimension culturelle et artistique du Congo et la seconde porte sur les perspectives qu'offre l'implication d'une telle dimension dans le développement global du Congo et des enjeux et de l'impact de la culture dans l'élaboration d'une «mémoire collective » nationale et moderne, ouverte à la créativité contemporaine.

\section{De la profondeur préhistorique aux premières manifestations culturelles et artistiques}

Les âges de la préhistoire du Bassin du Congo (RDC et Congo-Brazza) commencent à être connus, définis et classés. Dès 1935, Jean Colette, africaniste belge, tente d'établir la première chronologie de la préhistoire congolaise. Mais c'est tout au début des années 1980 et 1990 que certains travaux, particulièrement ceux de Pierre de Maret, Raymond Lanfranchi, Bernard Clist, Aimé Manima, et récemment Abraham Constant Ndinga Mbo, ont pu établir l'âge des industries préhistoriques du Congo (Obenga 1989). La plupart des techniques issues de ces industries recèlent des gisements de surface attribuables au tshitolien. Suivant toutes ces indications archéologiques (de Maret 1989), on peut aujourd'hui affirmer que plusieurs hominidés et espèces humaines ont occupé, parcouru, marqué l'espace actuel du Congo : sur la base des vestiges lithiques des outils récoltés, il est fort possible de dessiner les types d'hommes préhistoriques qui ont été les initiateurs, les créateurs des outils récoltés, leurs âges respectifs, les fondements approximatifs de leur milieu culturel. À cause de la présence de l'outillage lithique typique de la Pebble Culture et d'un certain nombre d'indices ostéologiques faisant signe à la présence de l'Homo habilis, ainsi que de la proximité du Bassin du Congo avec les régions de l'Afrique australe et de l'Afrique orientale, il peut être établi que l'occupation humaine du Congo remonterait aux âges australopithéciens, c'est-à-dire à plus d'un million d'années. Ainsi donc, on peut dire que, suivant les différents âges archéologiques que le Bassin du Congo a connus, le tshitolien est, (dans presque toute l'Afrique centrale), l'âge le plus "récent" de sa préhistoire, celui où s'amorce son processus de néolithisation. Sur diverses collines dominant les grandes vallées du Niari ou du Congo et de ses affluents, on trouve les outils caractéristiques de cet âge. De facto, les armatures tshitoliennes raffinées et spécialisées que l'on trouve ici conviennent parfaitement à une culture de la pêche, de la cueillette et de la chasse aux moyens et petits animaux ainsi qu'aux oiseaux. Ce caractère évolué, ces gisements superficiels, l'utilisation magique actuelle relativement fréquente d'autre part de cet outillage tshitolien accréditent l'hypothèse de sa date récente (Buakasa 1989). S'en suit alors la classique protohistoire des métaux, cuivre et fer, argent et plomb. Désormais, il est admis que cet âge des métaux est celui par lequel se sont affirmés les peuples dits «Bantous" dont les descendants sont regroupés en quatre grandes familles linguistiques: Teke, Kongo, Ngala et Maka. 
10 S'il est convenu de reconnaître que ce sont ces Bantu qui constituent le plus grand groupe des habitants de l'espace culturel du Bassin du Congo, il y a lieu cependant de dire que les Twa (Pygmées) furent les "premiers» hommes à s'installer dans cet espace. Et c'est au fur et à mesure que les Bantu prennent possession des lieux à partir desquels s'est dessiné historiquement et culturellement l'actuel territoire du Congo (Vansina 1989). Dès lors, l'affirmation de l'identité culturelle du Congo ne répond pas à un simple besoin de se singulariser. Elle n'est, ni une simple proclamation du droit à la différence, ni le signe d'une crise politique ou d'un désarroi économique, pas plus qu'une réaction pathologique au traumatisme des effets pervers du colonialisme. Elle n'est ni réflexe d'autodéfense face à l'agression de la culture technologique européenne issue de la nouvelle mondialisation et de la globalisation de l'économie du marché, ni processus de purification destiné à détruire les germes de la diversité culturelle, base de toute démocratie authentique et de l'instauration d'un État de droit républicain et laïc.

11 C'est par conséquent l'esquisse des traits les plus caractéristiques de la personnalité culturelle et artistique congolaise plurielle qui peut servir de creuset au surgissement d'une pensée congolaise moderne efficace. Car la circulation des idées et des biens culturels qui, à travers les âges, entretiennent les différentes régions du Congo et d'autres pays d'Afrique centrale, est amplement attestée. Frontières naturelles avec la République démocratique du Congo, l'Angola, la République centrafricaine, le Gabon et le Cameroun, cette circulation en dépit des obstacles naturels et des conflits en tout genre s'est étendue dans tous les sens, et a participé à l'éclosion et aux différentes créativités artistiques, spirituelles, littéraires et façonné les imaginaires anthropologiques de l'homme congolais. Le fleuve Congo lui-même, ainsi que l'Océan Atlantique ont représenté un lien naturel entre les différentes régions du Congo et les autres parties de l'Afrique et du monde, installant de fait le Congo dans les circuits de la modernité et du développement durable. Aussi se donne à lire dans le déploiement de la créativité congolaise contemporaine, la conjonction de l'esprit de cette culture plurielle avec celui du développement arrimé à la modernité.

12 Car un développement durable est toujours nourri constamment de l'intérieur par un esprit culturel, un idéal, une philosophie, une esthétique, une éthique et une politique, c'est-à-dire prosaïquement par une pulpe vivante d'idées, de croyances, d'ambitions, de thématiques inlassablement imaginées et inventées qui propulsent le développement dans le temps et dans l'espace. C'est ainsi qu'il faut comprendre pourquoi les arts, les lettres, et tout ce qui participe à l'élaboration des formes et expressions matérielles et immatérielles donnent un sens nouveau à la vie de l'esprit culturel du Congo déroulant ainsi sa politique culturelle.

\section{Tendances historiques des formes et expressions artistiques du Congo}

Dans la mise en œuvre d'une mémoire collective efficace au Congo, en tenant compte du poids historique de ce pays dans le dégagement d'une rupture entre le passé et le présent, la ville de Brazzaville apparait comme le point de mire d'une expérimentation originale. Car, à la veille de l'indépendance du Congo, Brazzaville fut à la pointe d'une nouvelle expression culturelle et artistique issue des pratiques traditionnelles de 
l'arrière pays en confrontation avec celles dérivées de l'action culturelle urbaine de l'administration coloniale française.

En effet, l'explorateur français d'origine italienne, Pierre Savorgnan de Brazza a eu son nom donné à la métropole du Moyen-Congo et du Congo : Brazzaville, sur la rive droite du fleuve Congo, en pays téké où existait déjà le village Mfoa, sous l'autorité d'un chef politique et spirituel qui obéissait lui-même au roi résidant à Mbé (Ibalico 1955). Le pouvoir du chef de Mfoa s'étendait sur le puissant fleuve, en concertation avec les autres chefs téké de la rive gauche qui commandaient les ancrages de Ngobila, de Kitampo (Kintambo), jusqu'aux hauteurs du mont Amba (emplacement actuel de l'Université de Kinshasa, ex-Lovanium) et de Ngalieme ou Ngaliema. Il y avait deux grands marchés : Maya-Maya dans la plaine de Ngamaba (actuel aéroport international de Brazzaville) sur la rive droite, et Untsaya (ibid.) ${ }^{1}$ (d'où Kinshasa en phonation européenne sur la rive gauche). En fait le mot Untsaya veut dire en téké échange, et Maya-Maya signifie dans la même langue va-et-vient.

La vie, le trafic, le commerce, la navigation, la pêche, les échanges de toutes sortes liaient ainsi Mfoa et Untsaya hier, comme aujourd'hui Brazzaville et Kinshasa (exLéopoldville). Brazzaville ne peut pas vivre sans échanger avec Kinshasa, et vice-versa, ceci depuis bien avant la colonisation. La préhistoire des deux rives révèle des relations, au temps néolithique, entre les habitants de la rive droite et ceux de la rive gauche. C'est dire la profondeur temporelle des solidarités qui unissent Brazzaville à Kinshasa.

Dans les temps coloniaux et postcoloniaux, Brazzaville aura son histoire culturelle propre. Celle d'une ville glorieuse, aux nombreux monuments et aux symboles historiques et politiques toujours vivants. Ceux-ci doivent être intégrés dès le départ dans l'élaboration d'une politique culturelle harmonieuse. Parmi les monuments historiques les plus évocateurs de Brazzaville, on peut citer : la cathédrale à vitraux du Sacré-Cœur de Jésus bâtie par $\mathrm{M}^{\mathrm{gr}}$ Prosper Augouard, la basilique-souvenir Sainte Anne du Congo, œuvre gigantesque du R.P. Charles Le Comte, la Case du général de Gaulle, l'immense stèle souvenir de Pierre Savorgnan de Brazza surplombant le fleuve Congo, la colossale statue du gouverneur général Félix Éboué devant le stade qui porte son nom.

17 Ces choses de pierre et de bronze, puissantes, grandioses, spectaculaires, enracinent ainsi Brazzaville dans le temps historique. Elles lui donnent son visage, son âme et sa renommée. En se promenant à Brazzaville, on va nécessairement à la rencontre de l'histoire, celle de grands hommes qui ont laissé l'exemple du dévouement, de la patience, du devoir, de l'honneur et de la gloire. Brazzaville est une cité, certes, mais une cité bâtie dans l'éclat de la lumière équatoriale et verdoyante de sa superbe robe de la forêt de la patte d'oie. On l'appelle ainsi « Brazza la verte »!

Ville historique, Brazzaville est aussi une ville politique qui a ses symboles et ses mythes puissants à l'instar de grandes figures telles que Matsoua, de Gaulle, Félix Gérald Tchicaya, Jacques Opangault, Fulbert Youlou. Brazzaville est fière de ces personnages qui ont marqué et marquent encore aujourd'hui la conscience politique des Congolais. Ces figures politiques devenues de véritables icônes historiques traduisent aussi l'activisme culturel et «militant» de la jeunesse congolaise. Car la jeunesse brazzavilloise a toujours été "activiste», évoluant en associations socioculturelles et militantes. Pendant longtemps, la jeunesse de Brazzaville, à l'image des jeunes de « Poto-poto », n'a pas connu le «tribalisme » ou le « régionalisme ». Seuls 
comptaient pour elle le débat d'idées et les stratégies de lutte pour la liberté au sein des cercles culturels de Poto-poto et de Bacongo. Le magazine Carrefour des Jeunes, animé par Sylvère Tsamas et ses amis de tous les horizons du Congo, est un témoignage historique concret de l'éveil à la fois culturel et politique de cette jeunesse toujours en quête de nouveauté et d'originalité dans les années 1950-1960.

Ville historique, politique, Brazzaville est aussi et surtout une ville culturelle et intellectuelle, possédant jadis des librairies, des bibliothèques et des revues de belle qualité et de bonne facture. Car c'est encore la métropole congolaise qui a initié toute l'Afrique équatoriale française (AEF) aux belles lettres grâce à la célèbre revue Liaison, fabriquée, éditée et animée à Brazzaville par Lomami-Tchibamba (RDC), Patrice Lhoni, Leyhet-Gaboka, Mambéké-Boucher, Jean Malonga, Antoine Letembet-Ambily, Galin Douate (RCA), etc.

Le Lycée «aéfien » Pierre Savorgnan de Brazza, si magnifique autrefois, a envoyé en France ses premiers élèves pour des études universitaires, plusieurs années avant l'indépendance du Congo en 1960. Seul le mérite prouvé comptait. La discipline stricte était sentie plutôt comme un bien par tous les élèves ; il fallait travailler d'arrache-pied pour prendre part au Concours général de la métropole et se voir bien classé lors de la distribution des prix en fin d'année. Les aînés servaient de modèles aux cadets dans les études et les examens. La part du Lycée Savorgnan de Brazza et du Lycée Chaminade (confessionnel) dans la formation des cadres est irremplaçable.

21 Culturelle, Brazzaville très tôt s'est installée dans le cycle de la mode et de l'élégance. Les enfants de Poto-poto et ceux de Bacongo, les somptueuses filles de ces deux vieux quartiers de la cité, ont des " amicales », des " associations », des «fraternités » et des "sororités " qui existent depuis plus d'un demi siècle. L'association féminine « La violette " a traversé le temps, mais la grâce, l'élégance, la distinction, le raffinement demeurent comme au bon vieux temps des célèbres bars-dancings tels que chez «Faignond", "Bankaitès ", " Macedo » ou les "Cataractes ». Toutes ces indications montrent que Brazzaville est une ville où il faut savoir cultiver les relations humaines. Les hommes et les femmes authentiques de Brazzaville déconsidéraient ceux ou celles qui ne tenaient pas à l'honneur de la parole donnée. Toutes les ethnies et toutes les générations se côtoyaient autrefois à Brazzaville dans l'amour, la solidarité, l'entraide, le secours mutuel, particulièrement lors des mariages, naissances, baptêmes ou décès. Des traditions existent, culturelles, artistiques, artisanales, culinaires, vestimentaires, musicales, photographiques, littéraires, ludiques, etc. Par conséquent, Brazzaville a toujours célébré, à sa manière, selon son style de vie et sa cadence historique le beau, l'élégance, le bien, l'amitié, la solidarité, l'intelligence, l'hospitalité.

22 En effet, par son hospitalité légendaire, Brazzaville est une ville extrêmement cosmopolite. La notion d'" étranger » y est inconnue, car depuis toujours cette ville vit en phase avec sa sœur jumelle Kinshasa. À Brazzaville, Noirs et Blancs, Tchadiens, Centrafricains, Camerounais, Gabonais, Angolais, Sénégalais, Maliens, Guinéens, Mauritaniens, Béninois, Nigérians, Ivoiriens, etc., vivent et ont toujours vécu en symbiose et en harmonie. Et ensemble, ils partagent parfois les mêmes souvenirs, établissent de merveilleuses relations et tressent parfois dans une complicité inouïe des choses agréables à voir, à entendre, à savoir et à savoir faire.

Les lieux, les squares, les monuments, les vieux édifices coloniaux, les dancings célèbres, les arbres, les bosquets et les avenues, constituent autant de repères pour les souvenirs et des étrangers et des Congolais qui habitent cette ville. Comment ne pas les prendre 
en compte quand il s'agit d'élaborer une vraie "mémoire " collective, base de toute historicité singulière en vue de la construction moderne du Congo ? Glorieuse cité, face à une autre énorme métropole africaine, Kinshasa, Brazzaville dite la « verte » forme en fait une seule et même grande masse urbaine avec « Kinshasa-la-Belle » : le Pool Malebo (ex-Stanley Pool), majestueux, unit les deux capitales les plus rapprochées du monde. L'île Mbamou, autrement dite l'île-des-rôniers, bien qu'appartenant à la rive droite, semble prolonger à la fois Brazzaville et Kinshasa comme d'ailleurs l'île-au-diable, aux puissantes chutes du Congo, là où précisément le fleuve franchit d'immenses obstacles de pierres. Avec donc tous ces atouts historiques, politiques, culturels et touristiques, Brazzaville apparaît donc comme le point focal à partir duquel l'on peut élaborer et expérimenter une politique culturelle en République du Congo. Celle-ci s'appuyant sur l'ensemble des infrastructures en place doit déboucher sur la mise en route des nouvelles acquisitions technologiques et sur l'érection de grands édifices culturels sur le territoire national. Par conséquent l'élaboration d'une politique culturelle basée sur une loi d'orientation nationale ne peut que consolider le développement du Congo.

\section{Congo terre historique de la sculpture africaine}

Avant de présenter les grandes tendances des formes et expressions artistiques congolaises de la veille de l'indépendance à aujourd'hui, je dois rappeler qu'il a existé un art sculptural traditionnel, très ancien, considéré comme l'une des plus fortes expressions plastiques de l'Afrique noire (Bassani \& Fagg 1988 ; Cornet 1989). En effet, si l'on considère le volume des études déjà publiées sur les arts d'Afrique noire depuis leur installation dans les sillons de l'art et de littérature de l'Occident sous l'impulsion des cubistes, des surréalistes et des expressionnistes au début $\mathrm{du} \mathrm{xx}^{\mathrm{e}}$ siècle, on se rend compte que la connaissance de ces arts est désormais solidement constituée (Leiris \& Delange 1967; Rubin 1987-1991; Philips 1999; Grimaldi Forum 2005). Remontant jusqu'à la fin du Moyen-Âge et au début de la Renaissance européenne, la sculpture congolaise traditionnelle englobe une pluralité plastique qui traverse tout le territoire national. La statuaire téké, kwélé, kota, les «fétiches » à clous kongo, mbembé, les marottes en bois kyébé-kyébé mbosi-koyo, les masques tsayi, punu, yombé, les cuillers et salières en ivoire loango sont parmi les œuvres de «l'Art nègre » qui ont marqué la conscience esthétique euro-américaine du début du xxe siècle (Musée du Louvre 2000). On note cependant qu'il y a de profondes mutations sociologiques et esthétiques qui sont intervenues dans les structures sociales africaines post-indépendances dans la réception historique et esthétique de ces arts anciens et les arts africains modernes d'essence urbaine.

De ce fait, il n'y a pas à proprement parler, sur les plans symbolique et esthétique voire ontologique, de continuité ou d'héritage entre l'ancien art sculptural traditionnel et la sculpture congolaise contemporaine, essentiellement urbaine. Il n'y a ni possession, ni réappropriation et encore moins dénégation du sens esthétique ou plastique de cette sculpture congolaise contemporaine au détriment de l'ancienne. Autrement dit, la modernité de cette nouvelle sculpture n'est pas la version émancipatrice ou moderne de l'ancienne tradition sculpturale. Du point de vue historique et esthétique, il n'y a ni dépérissement, ni décadence, ni dépassement entre ces deux ordres plastiques. Entre les arts plastiques des aires culturelles congolaises précoloniales et ceux issus des enclaves urbaines indépendantes et post-indépendantes, il n'y a ni convergence ni divergence. 
Cependant, par les «ruses » de l'histoire, par le jeu inconscient ou conscient du miroir du passé sur le présent, on peut tout au plus induire quelques rémanences formelles de la sculpture traditionnelle dans la nouvelle. Donc, ni rupture, ni continuité entre ces deux expressions plastiques. Juste l'entrecroisement formel différé, dû à une reprise bien pensée et bien détournée de quelques motifs de la thématique sculpturale ancienne suivant des cas précis dans les tendances plastiques urbaines actuelles. Les canons sociologiques et culturels présidant à la mise en signe formelle et à la révélation $\mathrm{du}$ sens esthétique des formes et expressions plastiques traditionnelles ne sont plus opérationnels aujourd'hui (Bastin 1986). Les modes de réception, de circulation et de diffusion de ces œuvres ne relèvent pas non plus de l'économie d'un «marché de l'art " au sens occidental (Bidima 1997: 27-35). L'accès au travail du bois et des autres médiums, dans les sociétés traditionnelles africaines procède le plus souvent des pratiques initiatiques, cultuelles, sacrées, ésotériques (Mveng 1991). Se révèle un rapport ontologique avec les artefacts, qui est certes esthétique, plastique, mais dont la vocation est aussi d'ordre symbolique, sacré et d'établir un maillage entre le monde visible et le monde invisible (Obenga 1984). Dès lors, les lieux de révélation et de monstration de ces œuvres sont secrets et sacrés. Car ces œuvres ne sont pas toujours visibles pour tout le monde. Elles sont fortement «chargées » symboliquement. Elles incarnent les esprits des ancêtres ou des divinités de toutes sortes. Elles sont visibles suivant les grandes cérémonies qui rythment et marquent la vie sociale (Griaule 1938; Niangoran Bouah 1964).

En revanche, l'accès au métier de la sculpture contemporaine est le plus souvent une initiative personnelle. Ce métier est basé sur un apprentissage profane lié au plaisir de s'approprier un médium, de le travailler suivant sa propre conception, sans instruction esthétique relevant d'une autorité sacrée. Les marques de la signature sur le médium sont attestées et révèlent l'œuvre comme processus d'individuation. L'individuation est la marque, ici, de l'établissement de l'artiste congolais contemporain dans le microcosme urbain comme subjectivité signant son œuvre. Il s'agit donc de deux visions plastiques complètement différentes. Mais les artistes congolais contemporains, par les effets d'une certaine réminiscence historique et culturelle, ont su instaurer un dialogue incessant, entre l'ancienne sculpture et la leur. Car, du point de vue thématique, ces deux expressions sculpturales se retrouvent : elles célèbrent toutes les formes de vie de l'homme et de son univers.

\section{Tendances actuelles de la sculpture congolaise contemporaine d'essence urbaine}

L'analyse de la sculpture congolaise contemporaine montre que cet art actuel est essentiellement urbain. Nous serions tenté de dire que c'est une sculpture urbaine de Stanley Pool, rive droite du fleuve Congo. A priori, trois grandes tendances presque imperméables les unes aux autres s'imposent :

La première tendance très prolixe et conservatrice est celle des «descendants artistiques » de Mayola dont les représentants les plus célèbres demeurent Benoît Konongo et Grégoire Massengo. Cette tendance prend naissance dans les années 1930, précisément dans le village téké de Kingoma, appelé aujourd'hui "Massengo ", situé à une dizaine de kilomètres au nord de Brazzaville. C'est précisément dans ce village téké de Kingoma que Mayola va signer la première rencontre moderne congolaise entre 
l'imaginaire urbain et le bois. C'est lui qui initiera ses neveux Grégoire Massengo et Benoît Konongo au travail de la sculpture. En raison de son âge très avancé et de son éducation traditionnelle kongo "classique", ainsi que de ses connaissances personnelles acquises auprès des notables téké, il s'exerça dans un premier moment à reproduire des œuvres qui évoquaient la sculpture téké et mbembé qu'il connaissait fort bien. L'une des premières productions qu'il fit faire par son neveu Konongo, fut la reproduction d'une pipe traditionnelle mbembé qu'il possédait. La plupart des statuettes que Mayola exécuta avec l'aide de ses neveux Massengo et Konongo étaient en fait des commandes des chefs de terre téké et leur conception épousait la symbolique téké. Cette conception a longtemps inspiré l'œuvre de l'artiste Mayola et l'augmentation des commandes téké a joué un rôle déterminant sur les objets divers sculptés : coqs, oiseaux et surtout des pipes traditionnelles, très intériorisées, stylisées avec agencement d'une tête ouvragée ayant une ouverture servant de trou à tabac. Pour travailler ses œuvres, Mayola utilisait un bois nommé le "ngwala mumpala, mbaki ». Quelques années plus tard, à la fin des années 1940, Mayola quitta Kingoma, partit pour Léopolville (Kinshasa) sur la rive gauche du fleuve Congo. Il est malheureusement impossible aujourd'hui de trouver les œuvres de cet artiste dont la plupart furent « achetées » ou cédées aux chefs de terre téké.

Après le départ de Mayola, ses neveux Grégoire Massengo et Benoît Konongo continuèrent de faire fonctionner l'atelier de Kingoma. Cependant les commandes téké se raréfiaient alors que les statuettes votives et gémellaires d'inspiration téké perdaient de leur prestige auprès de Massengo. Les Téké avaient entre-temps «retrouvé » leur sculpteur «traditionnel » qui gardait l'anonymat; ce dernier retaillait le bois selon les "vrais » canons de leurs ancêtres. Grégoire Massengo procéda alors à une nouvelle orientation sculpturale de ses œuvres, suivant sa propre inspiration. Il élabora le système de représentation en bas-relief, qui existait déjà, mais qui, jusque-là, n'avait pas été systématisé comme style sculptural. Avec l'essence du bois appelé moungandji, très léger, facile à tailler, Grégoire Massengo mit au point, avec des matériaux maniables, de nouvelles possibilités plastiques à explorer. La trouvaille du système consistait à obtenir une homogénéité du fond en monochrome. Le noir était la couleur de prédilection dans le travail de Massengo. Un mélange d'huile au charbon donnait une pâte à l'effet escompté appliquée sur la surface traitée. Elle offrait ainsi l'impression d'une essence naturelle. Avec ingéniosité, l'artiste Massengo parvenait à élargir son champ d'action et diversifiait les thèmes choisis en conservant la représentation, travaillant des motifs zoomorphes (hiboux, cannes serpentines) ou anthropomorphes (pipes à têtes sculptées) et autres supports tels que des tabourets en bas-relief. Massengo voulait, par ce travail, atteindre des sujets stylisés, sculptés avec des interstices blancs faisant apparaître la beauté des motifs représentés et mis en relief. C'est ici qu'il faut signaler, qu'en dépit de cet effort d'élaboration sculpturale et en tenant compte des aspects très rudimentaires des outils du travail, les formes générales de ces statuettes ou de ces supports ne remplissaient pas toujours les conditions exigées ou les proportions relatives aux normes classiques d'orthodoxie sculpturale traditionnelle négro-africaine ou occidentale.

31 Ces objets représentaient tout simplement des évidences plastiques réussies, parfois inédites; ils témoignaient d'un travail artistique qui s'intercalait péniblement entre la sculpture traditionnelle téké/kongo d'essence rurale et la naissance d'un art urbain d'essence coloniale : ce qui confère un tant soit peu de valeur esthétique à ces œuvres. Ce travail révélait tout au plus que ces artistes congolais du début de l'ère moderne de 
la sculpture congolaise avaient un talent "inné » pour le métier du bois qui devait très tôt éveiller la curiosité des étrangers, particulièrement européens, artistes ou amateurs d'art de l'époque coloniale et susciter le désir de les «aider" à s'accomplir dans de nouvelles voies d'exploration plastique. Cet intérêt européen pour ces artistes "pionniers" était très ambigu: durant la période coloniale, on les indexait « d'artisans » et non d'artistes! Les artistes et mécènes européens adoptaient diverses attitudes correspondant à leurs préoccupations coloniales dominantes: soit, ils s'attachaient à "sauver " l'art traditionnel ancestral, menacé de disparition par les bouleversements politiques, économiques, socioculturels d'origine coloniale ; soit, ils se préoccupaient "d'initier» les "artisans" congolais à de nouvelles perspectives plastiques en rupture avec l'ordre sculptural ancestral ; ou encore ils cherchaient des formules « intermédiaires » destinées à concilier l'originalité négro-africaine avec les techniques modernes des formes plastiques d'essence occidentale.

C'est dans ce cadre qu'il faut situer la rencontre entre Benoît Konongo et l'architecte Roger Lelièvre connu sous le nom d'« Errel» vers 1945. Celui-ci, dans un souci de perfection technique lui fait reprendre plus d'une dizaine de fois une pipe qu'il voulait acquérir. À sa demande, Benoît Konongo quitta le village téké de Kingoma et s'installa quelques temps chez lui dans le quartier colonial européen de Brazzaville. Plus tard, il le fit engager dans la section des arts appliqués de l'École coloniale Édouard Renard de Brazzaville. Là, en compagnie de deux de ses cadets, Gustave Loumouamou et Édouard Malonga, il va initier d'autres jeunes Congolais au métier de la sculpture suivant les règles de cette école coloniale. Il va de soi que dans cette école, Benoît Konongo acquit une nouvelle expérience. Et, au contact des techniques modernes, il améliora sa propre technique sculpturale sur le plan de la finition et du rendu des matériaux.

Mais par rapport aux métiers d'art exécutés dans cette école, Benoît Konongo avait plus le statut de "manœuvre", "d'artisan » que d'artiste. Les œuvres produites étaient vendues aux expatriés européens par l'école qui concédait un salaire mensuel à Konongo et à ses élèves. Les oiseaux, les pipes, les têtes sculptées, les statuettes votives représentant des jumeaux constituaient les motifs de ce travail en bois de gwala.

C'est pendant son séjour à l'École Édouard Renard où il enseignait que l'architecte Roger Errel fit venir du Gabon la pierre de bigou. Ainsi, sur sa recommandation, Benoît Konongo exécuta pour le compte du gouverneur général Félix Éboué, l'une des premières œuvres congolaises en pierre de bigou représentant une jeune fille au torse nu. C'est également Errel qui lui fit découvrir le wengué, bois très prisé par les expatriés européens. Avant de quitter l'École Édouard Renard, juste à la fin de 1948, il exécuta sur commande du père Charles Leconte, chargé de l'office religieux à la basilique Sainte Anne de Brazzaville, trois statuettes votives représentant le Christ et la vierge Marie.

À l'École des arts appliqués Édouard Renard, comme chez Roger Errel où il travailla un moment sous sa direction, Benoît Konongo améliora son travail de sculpteur. A-t-il subi une influence plastique de la part de l'architecte français? Une transformation nette se fit sentir dans son travail d'élaboration sculpturale. Cela fut perceptible dans la géométrisation des motifs qui marqueront les œuvres qu'il exécutera au début des années 1950, lorsqu'il quittera l'École Édouard Renard, pour aller fonder son propre atelier de la rue Mayama, un quartier situé au nord de Brazzaville. C'est tout au début des années 1980 qu'il alla s'installer à Makélékélé, au sud de Brazzaville.

Parallèlement, Grégoire Massengo, qui était resté à Kingoma continua d'animer son propre atelier. C'est à l'occasion d'une vente de ses œuvres au quartier colonial 
européen qu'il fut découvert par le "commandant $»^{2}$ blanc de la ville de Kinkala, localité située dans la région du Pool au sud de Brazzaville ; celui-ci émerveillé par son œuvre, lui fit la proposition de décorer sa paillote. Il partit donc pour Kinkala et, avec l'aide de deux de ses élèves, décora tous les piliers en bois massif de la paillote. Par ce travail, il acquit une grande renommée dans toute la région du Pool et à Brazzaville; et quand il revint dans son village téké de Kingoma, son atelier prit de l'ampleur et, de facto, il l'agrandit. Il devint célèbre et le village Kingoma commença à attirer du beau monde colonial. C'est ainsi que la population environnante dénomma Kingoma en « Massengo »!

Dans la naissance des arts plastiques du Congo, particulière dans le dégagement urbain de la sculpture moderne, il est nécessaire de souligner la désappropriation culturelle coloniale qui fit perdre à Grégoire Massengo ses attaches d'origine kongo et téké. De plus en plus, en confrontation avec l'affirmation d'un certain nombre d'actions socioculturelles de l'administration, elles-mêmes maillées et érodées par les " impacts » culturels d'autres Africains en «situation coloniale » en Afrique équatoriale française, Grégoire Massengo, à l'instar d'autres artistes congolais et africains de cette époque, cherchait son chemin. De plus en plus, sa sculpture entamait une autre situation artistique inédite, complètement à la jointure du monde négro-africain ancien et de l'espace urbain déployé et imposé par l'administration coloniale française. Dès lors, Grégoire Massengo se mit à utiliser de nouvelles essences de bois dans son nouvel atelier de la rue Mayama et à déployer des thèmes résolument inédits dans l'imaginaire plastique congolais. Les objets créés accédèrent à une nouvelle esthétique plus affermie et gagnèrent une valeur plastique réelle et, en même temps, devinrent de véritables marchandises avec des prix variables. La plupart des commandes furent le fait des Européens qui travaillaient dans l'administration coloniale : rares furent les Congolais qui s'adonnèrent à ce commerce bien particulier. Le goût esthétique développé par la nouvelle installation plastique de Massengo correspondait plus à celui que souhaitaient les colons blancs. Il ne s'agissait pas ici de la naissance d'un marché de l'art au sens occidental du terme : il s'agissait en fait d'un espace « informel » qui permettait tout au plus à quelques amateurs d'artisanat dit « indigène » ou exotique d'acquérir des objets « primitifs » ou « curieux » selon l'expression de l'époque.

D'où l'importance de la recherche de nouvelles connaissances sur les essences de bois dont les spécificités établies donnèrent aux objets traités plus de brillance et de polissage. Le bois de fer appelé le wengué, le kambala, la pierre de bigou est de plus en plus utilisé. Et, vers les années 1956-1957, sur invitation de l'autorité administrative coloniale de Kéllé, localité située dans l'actuelle région de la Cuvette ouest, où il va travailler précisément pour une société d'exploitation minière, Grégoire Massengo découvrira l'ébène noire avec laquelle il exécutera quelques bustes, des masques et d'autres pièces majeures de son œuvre. Ce n'est que bien plus tard qu'il finira par utiliser le bois gris. En fin de compte, dans l'émergence de la sculpture congolaise contemporaine, Grégoire Massengo, à l'instar de Benoît Konongo dans la droite ligne tracée par Mayola, inaugure une œuvre plastique qui trouve ses racines dans l'entrecroisement des restes des traditions kongo/téké et les plissures culturelles et artistiques coloniales de la nouvelle ville de Brazzaville. Et qu'en tout état de cause, ces artistes occupent une place centrale dans l'histoire des arts plastiques du Congo moderne. 
39 Après cette première tendance de la sculpture congolaise contemporaine d'essence urbaine, apparaît une deuxième incarnée par Bernard Mouanga Nkodia. Celui-ci, sorti de l'atelier de maitre Giela de Ouenzé, où il resta de 1957 à 1959, fait la jonction entre cette première tendance et celle plus indépendante annoncée par l'œuvre sculpturale de Rémy Mongo-Etsion vers la fin des années 1970. Vers 1959, Bernard Mouanga Nkodia fut admis au concours d'entrée de l'Académie des beaux-arts de Saint Luc de Léopolville (Kinshasa). Là, il choisit l'option "sculpture sur bois"; il y passera quatre ans à acquérir les règles du métier dans un environnement influencé par la «modernité » de l'école dite « belge » (Badi-Banga 1977).

40 Cette formation pratique, essentiellement technique, se révélera propice pour le travail que cet artiste amorcera dès son retour à Brazzaville en 1964. Le retour sur la rive droite du fleuve Congo sera particulièrement intéressant car il sera embauché au centre forestier de Brazzaville comme enseignant-sculpteur, décorateur en design d'ameublement intérieur. La traduction variée et stylisée des œuvres de Mouanga Nkodia n'obéissait nullement aux caractères hiérarchiques, même si la tradition, dans son éventail des scènes de terroir, était respectée. Avec Mouanga Nkodia, on sent, on palpe des œuvres auxquelles il a su donner une vie intérieure. Marqué par une tendance occidentale classique, cet artiste a été fortement influencé par l'œuvre de Michel-Ange et Rodin. Il y a cette approche de la grâce de Rodin qui se dégage de ses œuvres et la force expressive des formes qu'il trouve chez Michel-Ange. Entre ces deux forces plastiques contraires, papa Mouanga, ainsi que l'appellent les intimes, a su par sa magie formelle transposer des attitudes plastiques qui marquent profondément la modernité de la sculpture congolaise contemporaine. Son écriture plastique est un véritable régal pour l'œil et l'esprit : ici se dessine une sculpture dont la limpidité et la finesse transmettent une forte émotion. Plusieurs fois primé sur le plan local et en Afrique centrale, papa Mouanga est considéré, à juste titre, comme l'un des plus grands artistes sculpteurs de sa génération. Son œuvre plastique compte parmi les plus prisées du Congo. À cheval entre la première tendance de la sculpture congolaise moderne d'essence strictement urbaine et la nouvelle tendance, il fait le lien entre les deux. Dominée par des formes géométriques filiformes et agréables à voir, la sculpture de papa Mouanga est une merveilleuse voie pour saisir le passage entre la tradition et la modernité dans l'émergence des formes plastiques congolaises post-indépendantes.

41 Dans cette deuxième tendance, à l'exception de l'œuvre de Bernard Mouanga Nkodia, il faut signaler également les œuvres des sculpteurs d'ivoire, descendus le long du fleuve Congo, influencés par l'art des miniatures des deux rives. Cette sculpture sur ivoire a pris naissance du côté des riverains bolobos, moye, ngangoulou, mbochis et téké de la localité de Makotipoko. Cette sculpture s'est affirmée entre 1950 et le début des années 1960.

42 La troisième tendance, très indépendante, expérimentale et complètement ouverte à la modernité des formes plastiques d'Afrique et du monde occidental est incarnée par l'œuvre de Rémy Mongo-Etsion. Cet artiste, à l'instar d'autres artistes plasticiens de sa génération comme Trigo Piula, Bill Kouélany ou Ndinga Eleizer, propose et professe une exploration plastique extrêmement fine des matériaux, méthodes et concepts nouveaux. Celle-ci se situe dans une dynamique d'interactivité et de dialogue avec les autres médiums pour un ressourcement évolutif et perpétuel, et aurait pris forme à la jonction des années 1980 et 1990. Sans être une tendance strictement homogène sur le plan plastique, bien distincte, cette tendance regroupe la plupart des jeunes sculpteurs 
congolais d'aujourd'hui. Elle trouve également ses échos dans l'œuvre plastique de Bill Kouélany - assurément la plus grande artiste peintre congolaise de ces vingt dernières années -, véritable hymne à la créativité plastique. Entre 1993 et 1994 cette tendance aborde le thème du mouvement compression/extension. Que ce soit sur le bois, sur la pierre, sur les métaux ou encore avec les couleurs, il y a chez Mongo-Etsion et Bill Kouélany une nette tentative de créer des formes plastiques nouvelles, complètement inédites. Assemblage des matériaux divers qui peut être une réplique d'une équation mathématique relative à la ligne fermée, à la ligne ouverte, symboles de la vie et de la mort. Opposition vie et vies vécues-vie/infinité-vies vécues-segments-vie close/vie ouverte-fini/infini-pénétration/dé-pénétration. Vies vécues, ce sont des segments; des sillons de la vie que chaque être vivant, humain, animal, végétal, minéral, trace, laisse dans la suite infinie du cosmos. Toute une phénoménologie de matériaux découpés, assemblés, désassemblés, ouverts, vides et pleins, se révèle ici. Même dans les dernières performances de Bill Kouélany il y a cette volonté manifeste, quasiment prométhéenne de visualiser à travers ces formes plastiques des mondes inconnus, inédits, des couleurs et des lignes qui font découvrir des horizons où l'invisible et le visible se côtoient sans se rencontrer si ce n'est pour ouvrir l'œil à des perspectives lointaines et merveilleuses. L'œil, au vu de ces matériaux « formés, déformés et reformés, n'arrête presque pas de voir et de revoir sans jamais trouver le point de vue et le point de fuite de sa vision: perspective sans point d'appui parce que toujours fuyant à travers les lignes pleines et vides de vie, naissance/mort/renaissance ».

La sculpture agencée à la peinture, à la photographie, à d'autres médiums, est comme une partition musicale, c'est-à-dire une suite infinie de sons, de couleurs, d'éclats étincelants de tous les bruits silencieux du monde. Le vide est silence, mais silence bruissant, silence gros de toutes les sourdines (bruits en fond sonore) de l'univers. Silence grouillant, remuant, comprimant/déprimant, libérant, criant à la fois ha! et ouf ! ou hum ! Comme la femme en état de grossesse attend dans le silence, gémissant pendant l'accouchement, les cris de l'enfant qui vient, qui arrive et qui annonce les couleurs et les sons de la vie. Telles apparaissent la sculpture et la peinture de Rémy Mongo-Etsion, de Bill Kouélany et de tous ceux qui sont dans leur lignée.

\section{Couleurs vives et merveilleuses du Congo}

44 Avec la naissance de l'École de Peinture de Poto-Poto, au croisement des années 1950 $\mathrm{du} \mathrm{xx}^{\mathrm{e}}$ siècle, la création plastique congolaise a pris de l'ampleur et s'est imposée au grand public international. La force créatrice ne s'est jamais démentie au fil des années, malgré quelques éclipses dues aux aléas de l'histoire, à un faible management de cette œuvre et à une régression de celle-ci. Depuis sa fondation par Pierre Lodds en 1951 sous le label du « Centre des arts africains ».

En effet, dans les années 1940 déjà, quelques peintres congolais ont leur propre atelier. Ils ont, pour la plupart, été initiés à la peinture moderne et possèdent, pour certains d'entre eux, une connaissance générale de l'histoire de l'art européen. Les plus connus d'entre eux s'appellent Jean Balou, Eugène Malonga, Faustin Kitsiba, Guy-Léon Fylla.

L'Afrique équatoriale française prend une part active à la Seconde Guerre mondiale grâce à Félix Éboué. En cette période de tourmente, Brazzaville a été élevée au rang de Capitale de la France libre par le général de Gaule. Il y a donc cette guerre mais, en même temps, les échanges sont nombreux, les artistes circulent, cherchant à faire 
fortune ailleurs que dans leur patrie d'origine. C'est ainsi qu'on retrouve à Brazzaville un Bela Borkema, peintre d'origine tchadienne qui venait de l'est du Congo belge où, grâce à Georges Desfossés, il avait appris les rudiments de l'art de peindre. De la même façon, Jean Balou, premier peintre congolais en date, voyage beaucoup sur le vaste territoire français d'Afrique équatoriale. Cette nouvelle forme d'expression artistique semble gagner quelques-uns comme on attrape une maladie incurable. La peinture à l'huile, des outils comme le pinceau sont choses nouvelles et l'artiste qui vient de les adopter apparaît déjà, dans sa société, tantôt comme une sorte d'hurluberlu, tantôt comme un magicien, un sorcier qui apprivoise les couleurs pour reproduire la réalité quotidienne et l'emprisonner sur une toile. Cette première fournée de peintres, ces pionniers ont la pleine conscience qu'ils font partie d'une nouvelle caste. Pourtant, il faut bien avouer que ce mouvement en train de prendre racine en Afrique est relativement tardif au Congo français. Au Congo belge voisin, les choses ont commencé dès avant 1925 et les expositions de "peinture nègre " en métropole attirent des curieux de plus en plus nombreux. Mais Brazzaville est une sorte de plaque tournante grâce à son accessibilité (le chemin de fer Congo-Océan relie Pointe-Noire et Brazzaville, tandis que le fleuve permet de naviguer jusqu'à Bangui) d'abord, mais sans doute aussi, grâce au prestige de la France. Cette effervescence donc, Brazzaville la doit en grande partie au succès d'un peintre d'origine camerounaise qui a choisi de s'y installer: Gaspard de Mouko. Ce dernier exerce une sorte de fascination sur les Congolais, surtout grâce à sa réussite sociale. En somme, au Congo de ces années-là, la peinture pouvait faire vivre son homme. Très en vogue à la même époque, les paysages font l'honneur des bonnes maisons. Les portraits sont également très demandés car ils sont un signe extérieur de richesse ; plus encore que le premier, ce dernier genre fait la fortune de ceux qui ont une grande maîtrise du dessin.

Militaire de carrière, Pierre Lods est en poste au Congo et, lorsqu'il quitte l'armée en 1949, il envisage de s'installer à Brazzaville comme peintre. Avec l'arrivée de Pierre Lods, les choses vont considérablement changer. On ne cherchera plus à reproduire la réalité, on va désormais la simplifier, la transformer et même, parfois, s'en écarter totalement. La méthode de Lods est simple : pas de cours, pas d'instruction; en un mot, pas de méthode. Il suffit de laisser courir le pinceau sur la toile, au gré de sa propre fantaisie, au gré d'une imagination qu'on se garde bien de brider au contact du monde extérieur. Tous les peintres que Lods trouve sur place sont à l'École européenne et c'est exactement le contraire de ce que recherche le peintre français. Pierre Lods souhaitait trouver, en effet, des peintres qui n'auraient jamais été au contact de l'art pictural de l'âme africaine. La légende, Guy-Léon Fylla, peintre et critique d'art, l'a fixée à travers un texte dont nous tirons l'extrait suivant:

«Pierre Lods, installé comme artiste peintre, prend à son service, en tant que personnel de maison, un jeune congolais, Félix Ossali, qui, nanti d'un sens de l'observation tout naturel, le regarde travailler du coin de l'œil en même temps qu'il s'occupe de ses travaux ménagers. Il est frappé par les couleurs avec lesquelles son patron joue à longueur de journée [...].

Quelque temps après, Ossali décide de faire comme son patron. Un matin, Pierre Lods, ayant abandonné comme d'habitude sa palette garnie de plusieurs peintures, s'en va faire quelques courses. À son retour, il surprend Ossali, pinceau en main, étalant sur un morceau de papier quelques couleurs de la palette, réalisant ainsi une véritable symphonie sauvage mais combien originale et frappante. Monsieur Lods, se gardant bien de le déranger, est émerveillé. Dans l'insouciance, Ossali continue son gribouillage jusqu'au moment où il s'aperçoit de la présence de son patron. Pris de panique, il tente de prendre la fuite, mais Pierre Lods le rattrape au 
bon moment et, tout heureux de trouver ce qu'il cherchait, le rassure, le met en confiance tout en le priant de poursuivre et d'achever son œuvre. Très peu rassuré, Félix Ossali continue, la mort dans l'âme, à manier la couleur jusqu'au moment où Pierre Lods lui demande d'arrêter, trouvant que l'œuvre parlait déjà de manière suffisante. Félix Ossali devint ainsi le premier élève de Pierre Lods [...] ».(Fylla 1994).

Il faut croire que Lods avait fait siennes toutes les grandes idées de fin de siècle qui débouchèrent sur la crise de la conscience européenne en matière d'art et surtout en peinture. Crise qui aboutit à une lente et progressive subversion du Réel - pris comme mimesis du sujet ou de l'objet représenté -, au profit d'une prise en compte de plus en plus prégnante dans son travail, du caractère aléatoire, variable, relatif, de ce Réel. Sans compter qu'aux difficultés liées à la perception pourrait s'ajouter, va s'ajouter la mise à nu, l'intrusion d'un imaginaire personnel désormais sanctifié par une manière de phénoménologie liée à la connaissance créative. La vogue de la psychanalyse ne fera d'ailleurs qu'amplifier ce mouvement et, d'une certaine manière, le parachever.

On admet généralement, pour des commodités de classement à la fois chronologique et esthétique, la succession de trois générations de peintures à l'École de Poto-Poto.

C'est ainsi que le style dit « Mickey » est considéré comme le premier style dominant de l'École. Celle-ci a d'ailleurs acquis sa renommée grâce à cette manière héritée sans doute des formes caricaturales des dessins animés du cinéaste américain. Voici d'ailleurs ce qu'en dit le poète et critique d'art Jean-Baptiste Tati Loutard (2003: 87) :

«L'École de peinture de Poto-Poto a connu plusieurs styles mais le plus marquant fut le style "Mickey". Inauguré par Félix Ossali, ce style se caractérise par des formes schématisées, rudimentaires. Les formes sont réduites à l'essentiel et rendues expressives par la fantaisie des mouvements, d'où le nom de "Mickey", par référence aux dessins animés du cinéaste Walt Disney. Ce sont en général de petits tableaux qui exaltent la vie africaine traditionnelle, à travers les scènes de chasses, de pêches, de marché, de guerre, de danse.

On a plusieurs fois relevé la ressemblance de cette forme d'art avec l'art rupestre du Tassili et de l'Afrique du Sud. Les personnages essaiment un espace sans troisième dimension. La grande vogue des "Mickey" se situe entre 1950 et 1954. C'est par ce style que l'école de peinture de Poto-Poto a été le mieux connue dans le monde. Il reste encore quelque chose de ce graphisme pictural chez des peintres comme Gotene dont l'art n'est pas sans parenté avec précisément l'art rupestre. Il suffit de voir son tableau "la caravane des porteuses" et "la théorie des jeunes filles" de la caverne de jeunes filles (Ravin de Tsisab) pour s'en convaincre. »

51 La toute première génération de la peinture moderne au Congo, celle des pionniers, n'est pas directement liée à l'École, mais a eu une influence sur les premiers élèves de Pierre Lods, dès 1951. C'était, on l'a vu, l'anti-modèle: ce dont il fallait s'écarter artistiquement. Ne serait-ce que pour cela, les pionniers eurent une certaine influence sur la première génération des peintres de Poto-Poto. Le fameux style " Mickey » n'est lié au Centre des Arts africains (premier nom de l'École de Poto-Poto de Pierre Lods), que parce qu'il permet aux disciplines de naître à l'art sans être à l'École de l'Occident, en ayant leur identité propre. Avec la naissance de l'École donc, commence la deuxième génération des peintres congolais. La troisième sera constituée par les nouveaux élèves du Centre des Arts, eux-mêmes pris en charge par les premiers élèves de Pierre Lods. Quelques-uns parmi ces nouveaux élèves deviennent très vite indépendants. Le plus célèbre, mais aussi le plus doué d'entre eux, s'appelle Gotene. Pierre Lods a beaucoup de mal à le diriger, à discipliner son tempérament par trop libertaire ; en un mot, à brider son imagination échevelée. Au bout de deux mois de rapports plutôt orageux avec le "maître ", l'élève finit par claquer la porte de l'École pour aller ouvrir son propre 
atelier où il va inaugurer un style pictural parmi les plus originaux de l'Afrique noire. Aujourd'hui, Marcel Gotene est considéré comme le plus grand artiste peintre congolais vivant.

Les « Mickey ", scènes de genre, racontent la vie quotidienne de personnages sur une toile. On voit la ménagère piler du saka-saka, tandis que le joueur de tam-tam semble donner le la à des danseurs. Plus loin, d'autres personnages dans d'autres attitudes, avec d'autres occupations, reproduisent le menu d'un quotidien que les jeunes peintres répètent à l'envie.

53 Plus tard (milieu des années 1960), le style s'affinera, mais les scènes de chasse et de pêche auront à peine été remplacées par des scènes de marché, des foules bigarrées (Ondongo) et, beaucoup plus tard (années 1970), par un inextricable enchevêtrement de formes et de couleurs, rappelant quelquefois les masques de l'Afrique traditionnelle.

Ce changement de sujets correspond sans doute à une très forte urbanisation due à un exode rural massif qui, par ailleurs, s'explique moins par le développement économique des villes (notamment Brazzaville et Pointe-Noire) que par les mentalités laissées par une colonisation subtile des esprits, à travers cette recherche éperdue de la reproduction du modèle colonial. En effet, la ville exerce une telle fascination sur les Congolais que, très vite, telle une pieuvre aux multiples tentacules, elle vide la campagne de ses bras les plus vigoureux. Elle concentre tous les fantasmes, tous les rêves de fortune et de gloire habitant la jeunesse et que le cinéma (notamment les films d'espionnage américains) fait miroiter. Paradoxalement, l'effervescence de la ville est absente des toiles. Le nu semble également un sujet tabou.

Au milieu des années 1960, l'École s'affirme comme étant le vivier le plus important de peintres congolais. Les premiers élèves sont devenus des maîtres, chacun ayant développé son propre style.

D'exposition en exposition, l'École apparait, dès les années 1950, selon le bon mot de Ballis (directeur du Centre des Arts de Prétoria), comme « l'événement pictural le plus important de l'Afrique d'aujourd'hui ».

Avec des artistes comme Iloki, Okola, Ossiete, Ondongo, Thango, Ouassa, Ossali, Zigoma, Ngavounka, Ntuta, Bandila, Tatty, Iloki (fils), Mangwanza, Boboma, Ted Elongo, cette École a connu des fortunes diverses. Et avec des artistes indépendants de la trempe d'Eugène Malonga, Guy Léon Fylla, Gotene, Kitsiba, Noël Letolo, Ndinga Hilarion, Mokoko, Hengo, Mounkala, Trigo Piula, Rémy Mongo-Etsion, Elenga, Bill Kouelani, Balonga, Tondo-Ngoma, Gastinaux, etc., cette œuvre plastique, sous toutes ses formes et couleurs s'est nourrie de toutes les sensibilités culturelles du Congo.

La sculpture congolaise contemporaine a été inaugurée dès les années 1930-1940 par Mayola dont le style sera repris et développé par Benoît Konongo et Grégoire Massengo, sculpteurs congolais bien connus en Afrique. Cette première tendance sera suivie par Bernard Mouanga Nkodia issu de l'atelier du maître Étienne Giela. Avec Babindama, Mbia, Mongo-Etsion, et Mouanga-Nkodia, la sculpture congolaise aborde le gigantisme monumental. Enfin celle de ces dix dernières années, attestée par les œuvres de Molvy Nzonzy, Leandre Itoua, Nicolas Bissi, Eugène Abdon Nsonde, Eliezer Josias Dinga, Clotaire Samba, Séverin Kinzonzi-Mouendo, Béa Matt, Pass'Iza. Les couleurs chatoyantes, chaudes, grouillantes, l'expression volontaire ou intuitive de cette peinture et de cette sculpture sont d'une force inouie ouvrant à des imaginaires merveilleux, tourmentés, mais jamais pessimistes. C'est à quelque chose près ce qui 
distingue également l'univers de la littérature congolaise moderne. Euvre remarquable, originale, la littérature congolaise est l'une des plus intelligentes et puissantes de l'Afrique subsaharienne. Constituant une véritable "phatrie», les écrivains congolais excellent dans le roman, la nouvelle, la poésie. De Jean Malonga à Alain Mabanckou, en passant par Tchicaya U Tam'Si, Letembet-Ambily, Tati-Loutard, Guy Menga, Henri Lopes, Sylvain Bemba, Tchtchele Thivela, Théophile Obenga, Sony Labou Tansi, Emmanuel Dongala, L. Congo-Bemba, Biyaoula, Boniface Mongo, Nené Amélia, Marie Léontine Tchibinda, Aimée Mambou Gnali, Maxime Ndébéka, Jean-Blaise Samba-Bilombo, la littérature congolaise de ces cinquante dernières années constitue assurément l'une des plus belles pages de l'écriture de l'histoire culturelle congolaise contemporaine. Elle est foncièrement habitée par le Congo sous toutes ses coutures, ses splendeurs et ses malheurs. Elle est rageuse dans sa singularité, pugnace dans sa thématique et décapante dans son humour et sa gouaille. Elle est inclassable et unique dans le paysage littéraire africain.

CEuvre exaltante, célébrant la vie, sous forme de réflexions, de sentiments, de sensations, la littérature congolaise moderne de ces cinquante dernières années, par ses tonalités et ses saveurs nouvelles, a ouvert grandes les frontières imaginaires du Congo au monde.

61 Célébrer la vie dans toutes ses dimensions, c'est aussi le credo de la musique congolaise de ces cinquante dernières années. En effet, les années 1950 sont, pour les musiciens du Congo des deux rives du Stanley Pool, celles de la réappropriation des rythmes des Antilles, des Caraïbes. Le mambo, le merengue, la mazurka, la biguine, la marche, le fox-trot, la rumba, le cha cha cha, le boléro, le "gévé » envahissent le marché phonographe congolais puis, par la radio naissante et les premiers bars-dancings, intègrent le patrimoine sonore du Congo par un brassage complexe d'éléments tirés du fond local, de la rencontre avec les Africains venus du Golfe de Guinée qui apportent le highlife. Et, au début des années 1960, la romance euro-andalouse prend pied dans l'installation des sons du Congo ; romance inspirée par le succès de Patrice et Mario, Georges Guetary, Luis Mariano, Tino Rossi, mêlée parfois à un simulacre du créole, de l'espagnol, qui meuble les loisirs des Brazzavillois dès le samedi soir.

C'est sans conteste Paul Kamba qui constitue la référence mythique de cette musique. Les autres pionniers de la musique congolaise moderne sont Dadet Damongo, Lebel Massamba, Guy-Léon Fylla. Suivront ensuite les ensembles négro-band, Bantous de la Capitale, Orphée Jazz, Cercul Jazz, Tembo. Les musiciens comme Essous, Nino Malapet, Ophélé, Opia, Demon Kazano, Loubelo De la lune, Franklin Boukaka, Célio Nkounka, Papa Noël, Jacky, Jojo, Marie Bella, Mammadou Ntouta, ont émerveillé plus d'un Congolais entre les années des indépendances et la «Révolution ». La période qui suit est exemplaire de créativité avec l'arrivée des musiciens comme Joseph Mulamba Mujos, Mbemba Pamélo (Pablito), Mountouari Côme Kosmos, Biyeli Gerry Gérard, Bitsikou Théo, Richy Siméon dans les Bantous. C'est dans la même veine que s'affirmeront des jeunes ensembles comme Mando Négro "Kwala-kwalakwa», Super Boboto, los Batchitcha, les Rumbaros, les Fantômes, Super Kwala-kwalakwa Sindza Koto-koto, Africa Mod «matata», Manta lokoka, Novelty, Super Tembessa, Mok'National de Kiwo. Et, au croisement des années 1970 et 1980, font surface des orchestres dits "amateurs" des élèves des lycées comme les "Techniciens", le "groupe rouge", ainsi que ceux des quartiers populaires à l'instar des "Bilengue Sakana». Viendront se greffer à cette panoplie, les Dzoï, le Peuple, Télé Music, les 
Anges. Depuis une vingtaine d'années ce sont Nzao, Extra-Musica, les Patrouilles des Star, le Brazzavillois Rapha Afara Mbouendzeki, Kingoli qui tiennent le haut du pavé.

\section{La créativité congolaise à l'épreuve de la guerre}

63 Avec le mouvement Recycl'Art amorcé en plein milieu des années 1990 et durant la période la plus sombre de l'histoire du Congo, la créativité congolaise moderne prend également un nouveau tournant.

En effet, ce mouvement fait le recyclage artistique des objets usés, disparates et « jetés » à la rue.

Sous la responsabilité de Nicolas Bissi, peintre sculpteur et grand animateur culturel, ce mouvement rassemble de jeunes artistes qui ont transformé toute une avenue du centre de Brazzaville en œuvre d'art.

Papito, le plus jeune du groupe, a moins de quinze ans. Anicet Malonga, Eugène Nsondé, Magloire Nzonzi, Serge Ouaboulé et Molvy Nzonzi n'ont pas encore trente ans. Ils sont peintres, sculpteurs et scénographes. Recycl'Art est le prétexte, le seul qui puisse unir ces artistes d'horizons si divers : confluence transversale, tel est le mot d'ordre de ce mouvement (Bissi 1998) ${ }^{3}$.

Pendant deux mois, en avril-mai 1998, ces artistes ont ramassé dans les décharges publiques, les chantiers, les garages et amassé boîtes de conserve, carcasses de réfrigérateurs, écrous, bougies de moteur, vaisselle cassée... Leur objectif : créer des œuvres d'art à partir des objets de récupération et les exposer « à même la rue » car, désormais, ils appartiennent à la rue. D'autant plus que ces objets sont les «témoins vivants ", non parlants, non humains, mais symboliques de la guerre civile qui a détruit la ville de Brazzaville en 1997.

Soixante jours d'imagination créative et de "folie positive » de la vie, opposée à la « folie négative » de la guerre et de la mort. À la pulsion de la guerre, génératrice de la mort, il faut opposer la pulsion de l'art qui est générateur de vie.

Pendant soixante jours, ces jeunes artistes ont pris d'assaut une grande avenue de Brazzaville avec des couleurs, des collages en procédant par la même occasion au recyclage de tout objet trouvé dans la rue en lui donnant une nouvelle dimension pour aborder et entrer dans le monde nouveau créé par ces objets usés.

D'une carcasse de réfrigérateur et d'un tuyau d'échappement de motocyclette naît un trompettiste ; un réservoir de moto, des amortisseurs d'automobile, des couvercles et des chaînes de transmission animent un couple dansant le "dombolo ", la nouvelle danse en vogue à Kinshasa et à Brazzaville. D'un chauffe-eau surmonté de quatre tuyaux de fer sort un éléphant dont la queue est un robinet qui déverse un jus de tangawis (plus connu sous le nom de jus de gingembre) très prisé par les Brazzavillois ; un simple carton de sucre devient un support plastique, au même titre qu'une toile; le charbon de bois, la farine de foufou, les feuilles de n'importe quelle plante, le kaolin, sont des piments utilisés par ces artistes. La rue ainsi «squattée » se transforme ellemême en œuvre d'art.

71 "L'art dans la rue ", qui est un autre concept de ce mouvement, indique tout au plus que la rue, à l'image d'une œuvre d'art, est une porte ouverte sur la vie, sur le monde. De ces objets usés, jetés et rejetés, cassés, concassés, déchargés, défaits et destitués de leur nature originale par le recyclage qu'ils subissent, ils accèdent à une nouvelle 
topologie d'être et à être. Ces objets deviennent autre chose et offrent à l'œil un monde inédit, un monde en attente d'un nouveau matin, d'une nouvelle aube. Ce qui a été l'objet usé, jeté, recueilli par la rue et recyclé par l'artiste devient autre chose en nous ouvrant grandes les portes de la vie, du monde, annulant ainsi «les forces négatives » de la folie, du temps sombre, crépusculaire de la guerre et de la mort, c'est-à-dire du non-être.

C'est en suivant ce credo que Rémy Mongo-Etsion, Bill Kouélany, Gatinaux, Trigo Piula, Claude Balonga, Eleizer Ndinga et tous ceux qui sont dans leur lignée font de la sculpture, de la peinture et de tous les autres médiums une célébration plastique de la vie, des vies vécues. C'est ainsi qu'il faut déchiffrer le sens nouveau de la créativité plastique congolaise contemporaine à l'aube du XXI siècle.

\section{BIBLIOGRAPHIE}

AKA-EVY, J.-L., 2006 L'image des arts d'Afrique noire dans le discours esthétique occidental moderne. De la Renaissance au temps de Picasso puis de "l'art primitif » aux « arts premiers », Thèse de doctorat, Paris, Université de Paris 1-Panthéon Sorbonne.

BADI-BANGA, N.-M., 1977 Contribution à l'étude historique de l'art plastique zaïrois moderne : fin XV siècle-1975, Kinshasa, Malayika.

BASSANI, E. \& FAGG, W., 1988 Africa and the Renaissance. Art in Ivory, New York, The Center for African Art and Prestel, Verlag.

BASTIN, M.-L., 1986 « Art sculptural de l'Afrique Bantu », Muntu, 4-5, CICIBA, Libreville : 135-163.

BIDIMA, J.-G., 1997 L’Art négro-africain, Paris, PUF (« Que sais-je ?»).

BISSI, N., 1998 « Recycl'Art : recycler, réutiliser, recréer », Lemba, 1, 15 mai.

BUAKASA, T. K. M., 1989 « Croyances et connaissances », in T. OBENGA (dir.), Les peuples Bantu.

Migrations, expansion et identité culturelle, Paris, L'Harmattan : 175-189.

CORNET, J., 1989 « L'Art dans la culture bantu », in T. OBENGA (dir.), op. cit., t. II : 415-420.

DAPPER, O., 1686 Description de l'Afrique, Amsterdam, Wolfgang, Waesberge, Boom \& van Someren.

DIOP, A., 1951 « Éditorial : l'artiste (noir) n'est pas seul au monde », in A. DIOP (dir.), L'Art nègre, Paris, Présence Africaine.

DIOP, C. A., 1954 Nations nègres et culture, Paris, Présence Africaine.

-, 1967 Antériorité des civilisations nègres : mythe ou vérité historique ? Paris, Présence Africaine. FU-KIAU KIA BUNSEKI-LUMANISA, A., 1969 Le Mukongo et le Monde qui l'entourait. Cosmogonie-Kongo (Nkongo ye nza yakun'zungidila: Nza-kôngo), Kinshasa, Office national de la Recherche et de Développement («Recherches et Synthèses no 1 »). 
FYLLA, G.-L., 1994 « L'École de Peinture de Poto-Poto et son impact dans la vie culturelle et artistique du Congo ", in Sanctuaire souvenir. Saint-Anne du Congo, Basilique de la liberté : 1943-1993, Congo.

GRIAULE, M., 1938 Masques Dogon, Paris, Institut d'Ethnologie-Musée de l'Homme.

GRIMALDI FORUM, 2005 Arts of Africa. 7000 ans d'art africain, Monaco, Grimaldi forum ; Milan, Skira.

IBALICO, M., 1955 « L'origine des Batékés d'Impila », Liaison, Organe des cercles culturels de l'AEF, 46 : 37-39.

LEIRIS, M. \& DELANGE, J., 1967 Afrique noire. La création plastique, Paris, Gallimard (« L’Univers des formes »).

DE MARET, P., 1989 « Le contexte archéologique de l'expansion bantu en Afrique centrale », in T. OBENGA (dir.), op. cit. : 118-138.

MUSÉE DU LOUVRE, 2000 Sculptures : Afrique, Asie, Océanie, Catalogue, Paris, Pavillon des Sessions, Musée du Louvre, Réunion des musées nationaux-Musée du Quai Branly.

MVENG, E., 1991 « La Symbolique dans l'art négro-africain », in T. OBENGA \& S. SOUINDOULA (dir.), Racines Bantu. Bantu Roots, Libreville, CICIBA : 193-224.

NIANGORAN BOUAH, G., 1964 La division du temps et le calendrier rituel des peuples lagunaires de la Côte d'Ivoire, Paris, Institut d'Ethnologie-Musée de l'Homme.

OBENGA, T., 1973 L'Afrique dans l'Antiquité. Égypte pharaonique-Afrique noire, Paris, Présence Africaine.

-, 1974 Afrique centrale précoloniale. Documents d'histoire vivante, Paris, Présence Africaine.

-, 1984 «Caractéristiques de l'esthétique bantu », Muntu, 1, CICIBA, Libreville : 61-97.

-, 1991 « Histoire du Monde Bantu », in T. oBENGA \& S. Souindoula (dir.), Racines Bantu. Bantu roots, Libreville, CICIBA : 121-150.

OBENGA, T. (dir.), 1989 Les peuples Bantu. Migrations, expansion et identité culturelle, Paris, L'Harmattan.

PHILIPS, T., 1999 Africa. The Art of a Continent, Munich-London-New York, Prestel Verlag. PRÉSENCE AFRICAINE, 1956 Numéro spécial (8-9-10), « Le $1^{\text {er }}$ Congrès International des Écrivains et Artistes Noirs ", Paris, Sorbonne 19-22 septembre,

-, 1959 Numéro spécial (24-25), « Deuxième Congrès des Écrivains et Artistes Noirs », Rome, 26 mars- $1^{\text {er }}$ avril, t. I, « L’Unité des Cultures Négro-Africaines ».

PROYART, Abbé, 1776 Histoire de Loango, Kakongo, et autres royaumes d'Afrique, Paris, C. P. Berton \& N. Crapart ; Lyon, Bruyset-Ponthus.

RUBIN, W. S., 1987-1991 Le Primitivisme dans l'art du 20 siècle, 2 vol., Paris, Flammarion.

SPENGLER, O., 1948 Le déclin de l'Occident. Esquisse d'une morphologie de l'histoire universelle, (trad. de l'allemand par M. Tazerout), Paris, Gallimard.

TATI-LOUTARD, J.-B., 2003 Libres mélanges. (Littérature et destins littéraires), Paris, Présence Africaine.

VANSINA, J., 1989 « Expansion et identité culturelle des Bantu », in T. OBENGA (dir.), op. cit. : 273-289. 


\section{NOTES}

1. Pour tout ceci voir également l'ouvrage collectif Brazzaville 1880-1980, Édition Publi, Congo, Brazzaville, 1980.

2. Expression locale de l'époque pour désigner le représentant de l'autorité coloniale dans les régions intérieures.

3. Notons que cette exposition a été soutenue par Danielle Adada, l'association "Brazza'Art », le projet culturel «Patrimoine franco-congolais» et le Centre culturel français de Brazzaville ; l'association « Nouvel'Art » en a assuré la coordination.

\section{RÉSUMÉS}

Résumé

Dans le dégagement de la pensée africaine contemporaine à l'aube des indépendances des pays africains francophones, les écrivains, les artistes et les intellectuels congolais ont joué un rôle essentiel. Il s'agit de retracer ici, aussi précisément que possible, les temps forts de l'émergence de la pensée congolaise post-indépendance.

Abstract

In the evolution of contemporary African thought at the dawn of independence of francophone African countries, writers, artists and intellectuals congolese have played a key role. In this article, we'll trace a accurately as possible, the highlights of the emergence of contemporary congolese post-independence thought.

\section{INDEX}

Mots-clés : Congo, arts, créativité, expressions artistiques, manifestations culturelles et artistiques, pensée congolaise, sculpture

Keywords : Congo, Arts, Creativity, Artistic Expression, Cultural Events and Artistic, Congolese Thoughts, Sculpture 